\title{
A Newborn with Barium Sulphate Aspiration Following Barium Contrast Study for Esophageal Atresia: A Case Report
}

\author{
Hristo Shivachev ${ }^{1}$, Yanko Pahnev ${ }^{1 *}$, Zdravka Antonova ${ }^{1}$, Edmond Rangelov ${ }^{1}$, Nadezhda \\ Tolekova ${ }^{1}$, Alexander Tcherveniakov ${ }^{2}$, Natalia Gabrovska ${ }^{3}$, Svetlana Velizarova ${ }^{3}$, Nikolay Yanev ${ }^{3}$ \\ and Dimitar Kostadinov ${ }^{3}$ \\ ${ }^{1}$ Pediatric Surgery Clinic, University Multiprofile Hospital for Active Treatment and Emergency Medicine 'N.I.Pirogov', Sofia, Bulgaria \\ ${ }^{2}$ University Hospital 'Sofiamed', Sofia, Bulgaria \\ ${ }^{3}$ Department of Pulmonary Diseases, Medical University - Sofia, Multiprofile Hospital for Active Treatment of Pulmonary Diseases 'St. \\ Sofia', Sofia, Bulgaria \\ *Corresponding author: Yanko Pahnev, University Multiprofile Hospital for Active Treatment and Emergency Medicine "N. I. \\ Pirogov"21, Gen. Totleben boul, 1606, Sofia, Bulgaria
}

\section{ARTICLE INFO}

Received: 蔧 July 21, 2021

Published: 蔧 July 29, 2021

Citation: Hristo Shivachev, Yanko Pahnev, Zdravka Antonova, Edmond Rangelov, Nadezhda Tolekova, et al., A Newborn with Barium Sulphate Aspiration Following Barium Contrast Study for Esophageal Atresia: A Case Report. Biomed J Sci \& Tech Res 37(4)-2021. BJSTR. MS.ID.006033.

\section{ABSTRACT}

We present a case of a newborn with barium sulphate aspiration following barium contrast study for investigation of suspected esophageal atresia. In the literature little has been reported on barium aspiration in childhood and there is no established protocol for treatment. We describe an operative method for treating baritosis - segmental to subsegmental bronchial excision, used in children with bronchiectasis.

Keywords: Barium; Aspiration; Tracheoesophageal Fistula; Subsegmental Bronchial Resection

Abbreviations: ICU: Intensive Care Unit; SIMV: Synchronized Intermittent Mandatory Ventilation Mode; FiO2: Fraction of Inspired Oxygen; PEEP: Positive End-Expiratory Pressure; CT: Computed Tomography Scan

\section{Introduction}

In the literature little has been reported on barium aspiration in children and there is no established protocol for treatment. We present a case of a newborn with barium sulphate aspiration following barium contrast study for investigation of suspected esophageal atresia. The treating modality includes an operative method for treating baritosis - segmental to subsegmental bronchial excision, routinely used in children with bronchiectasis. The case illustrates the importance of an accurate assessment, rapid diagnosis and prompt primary surgical repair. It is an example of a good long-term outcome due to the successful collaboration in a multidisciplinary team.

\section{Case Report}

A 1-day-old newborn (34 weeks gestational age) was admitted to our tertiary care hospital. The newborn was intubated after previously performed radiograph showing atretic proximal esophageal segment, presence of distal tracheoesophageal fistula and barium contrast in right inferior pulmonary lobe. (Figure 1) In the delivery room the baby was intubated, after manifesting signs of acute respiratory distress syndrome (ARDS) with multiple apnoic episodes. Pulmonary surfactant was administered. The baby showed sialorrhea and no ability for placing oral tube deeper than 
$10 \mathrm{~cm}$ from the lips. In the neonatal unit esophageal atresia was suspected and a barium contrast study was performed. The chest radiograph showed the above-mentioned changes. In our intensive care unit (ICU) the baby was still intubated and used synchronized intermittent mandatory ventilation mode (SIMV) with fraction of inspired oxygen (FiO2) 0.6, ventilation frequency 22 per minute and positive end-expiratory pressure (PEEP) $3 \mathrm{cmH}_{2} \mathrm{O}$. The oral tube was connected to a continuous suction device. Broad spectrum antibiotics were administered. On the next day the infant underwent surgery - the fistula was ligated followed by end-to-end esophageal anastomosis, due to sufficient distance between the two atretic pouches. During the postoperative period the baby failed extubation because of barium pneumonitis.

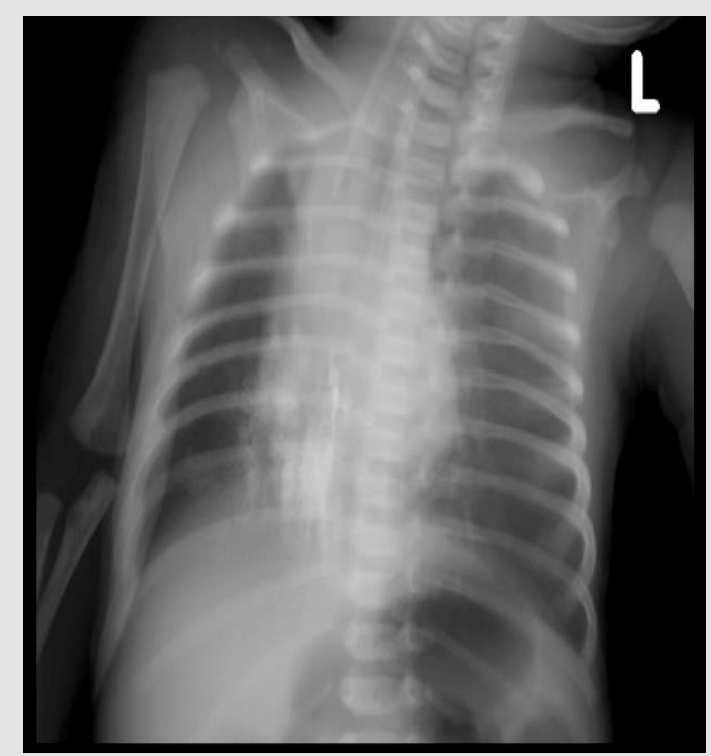

Figure 1: Preoperative Radiography.

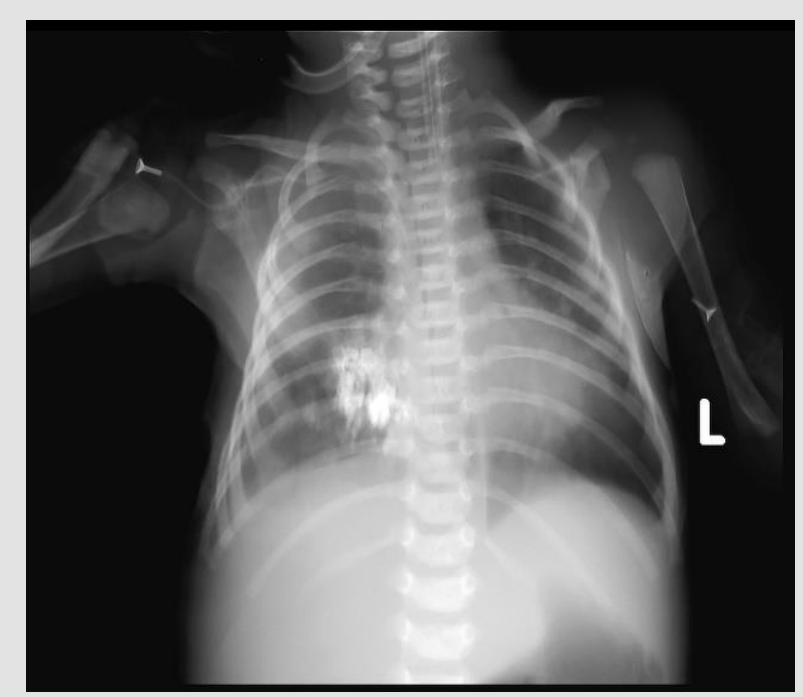

Figure 2: Before Rethoracotomy.

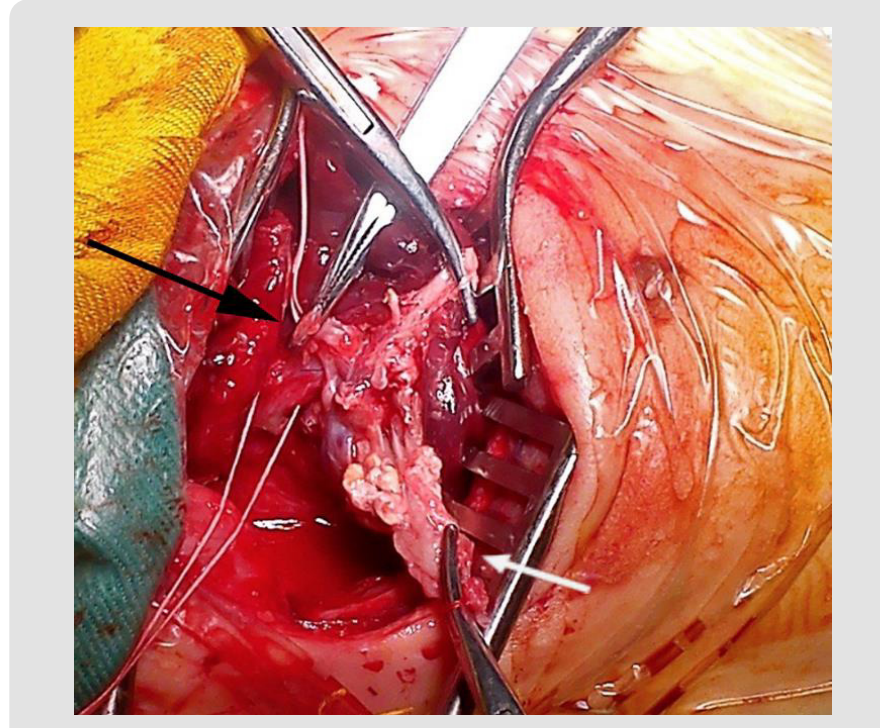

Figure 3: Intraoperative picture - white arrow - proximal segment; black arrow -subsegmental bronchi.

The excessive amount of foamy white secretion necessitated frequent tracheobronchial aspiration. On day 21 the patient underwent computed tomography scan (CT) which revealed deposition of the barium sulphate mostly in the right inferior lobe $6,9,10$ segments, and a right dorsal extrapleural thickening. On day 25 bronchoscopy was performed and endobronchial obstruction of the right inferior bronchus and hyperemia and edema of the other part of the tracheobronchial tree were found. Bronchoalveolar lavage was performed to reduce the obstruction with unsatisfactory effect. During the postoperative stay in the ICU there were several attempts to wean off the ventilator which led to fast desaturation $\mathrm{SatO}_{2} 88 \%$ and reintubation with increased ventilation parameters as follows $-\mathrm{FiO}_{2} 0.9$, ventilation frequency 42 per minute, PEEP 4 $\mathrm{cmH}_{2} \mathrm{O}, \mathrm{SatO}_{2}-96 \%$. (Figure 2) In order to reduce tracheobronchial secretion and the amount of barium deposition in the lung, we have decided to perform an operative procedure used for treatment of bronchiectasis in children. This method was described by Isakov, et al. [1] and consists of proximal ligation of the main bronchus and distal extirpation of segmental and subsegmental bronchi, thus preserving lung parenchyma and blood supply. (Figure 3) The postoperative chest radiograph revealed reduction of the amount of barium deposition in the right inferior lobe. Seven days after the rethoracotomy the baby was referred to a neonatology clinic intubated using $\mathrm{SIMV} \mathrm{FiO}_{2} 0.6$, ventilation frequency 20 per minute, $\mathrm{SatO}_{2}-97-98 \%$ for a continuous treatment. After one week the baby was successfully extubated. One month after the procedure the baby was breathing on her own, gaining weight and the chest radiograph showed bilaterally ventilated pulmonary parenchyma with significant reduction of the barium aspirate in the right inferior lobe. (Figure 4) The patient is scheduled for late follow-up assessments of complications such as fibrosis or others. 


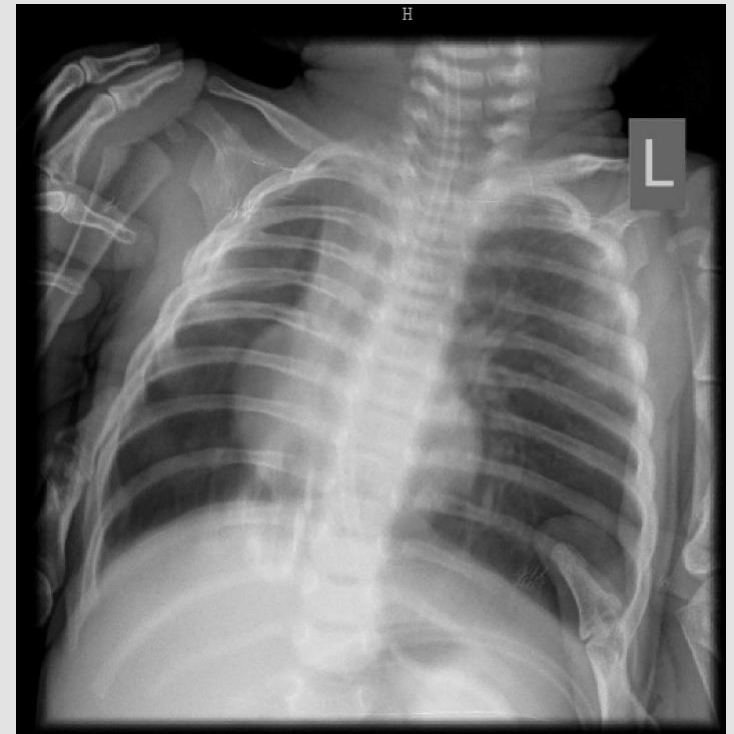

Figure 4: Post-operative follow-up.

\section{Discussion}

Aspiration of significant amounts of barium in infants is rare and there is no consensus on how to manage such complication. In the peer - reviewed literature different opinions about the effect of the barium aspirate on the pulmonary parenchyma are expressed. There are many reports of cases where no clinical symptoms, either acute or chronic, were found - 16 cases described by Wilson, et al. [2]; 19 cases (including 3 children) - by Shook, et al. [3]; over 200 human bronchograms - by Teixeria, et al. [4]. On the contrary, some authors describe acute respiratory distress, pneumonitis and even deaths [5-7]. Others report mortality rate associated with massive barium aspiration approximately $30 \%$ which may reach up to $50 \%$ depending on the initial patient's condition [8]. These different consequences can be explained by the influence of many factors like age, pre-existing clinical state, the concentration of the barium used $[7,9]$, the volume aspirated, and concomitant aspiration of gastric contents [10]. The distribution pattern of the barium within the tracheobronchial tree is determined by the patient's posture at the time of the study and clearance mechanisms such as cough, mucociliary escalator clearance, and cellular ingestion.

In our case we have assumed that the immaturity of the neonate's lungs combined with barium aspiration and the trauma from the thoracotomy performed for the concomitant esophageal atresia led to failure in weaning of ventilation and persistent pneumonitis. Some authors conclude that bronchoalveolar lavage is mandatory after barium aspiration [7]. On the contrary, others deny the lavage, because it may disseminate the barium further within the bronchoalveolar system [11]. We have tried to use fiberoptic bronchoscopy and bronchoalveolar lavage to reduce the amount of barium depositions with unsatisfactory result. In this case we propose an operative technique of treatment for baritosis -subsegmental bronchial resection, described in 1988 by Isakov as a method of surgical treatment for bronchiectasis in children [1]. The advantage of this method is that the residual pulmonary parenchyma can still be pneumatized via interalveolar paths. This also preserves the blood supply and avoids formation of residual cavity and chest deformity. Similar results can be seen in an experimental study in dogs - extirpation of bronchi, described by Zhonghua Wai Ke Za Zhi in 1996. In the control group of this study (with segmental resection) heavy destruction, consolidation and emphysema were observed in the adjacent lung tissues, while in the experimental group the residual lung tissues only had little wound reaction and no atelectasis.[12] Our case report shows that the barium will remain in the lung for a long period of time but is relatively inert.

\section{Conclusion}

There are many factors influencing the variety of consequences after barium sulphate aspiration. We believe that in cases without significant respiratory disturbances only supportive care is indicated. Patients with mild symptoms may benefit of therapeutic bronchoalveolar lavage. In those with respiratory distress and prolonged necessity of mechanical ventilation segmental to subsegmental bronchial extirpation should be considered.

\section{References}

1. Isakov IuF, Stepanov EA, Volynskii IuD, Vishnevskii AA, Schastnyi SA (1988) Extirpation and resection of the bronchi as one of the methods of surgical treatment of bronchiectasis. Grudn Khir Jan-Feb (1): 63-6.

2. Wilson J, Rubin P, McGee T (1959) The effects of barium sulfate on the lungs: a clinical and experimental study. Am J Roentgenol Radium Ther Nucl Med 82: 84-94.

3. Shook D, Felson B (1970) Inhalation bronchography. Chest 58(4): 333337.

4. Teixeria J, Texieria L (1959) Bronchography without oil and iodine - the use of barium as a contrast medium. Chest 36: 256-274.

5. Fruchter O, Dragu R (2003) A deadly examination. N Engl J Med 348(11): 1016.

6. Gray C, Sivaloganathan S, Simpkins K (1989) Aspiration of high-density barium contrast medium causing acute pulmonary inflammation report of two fatal cases in elderly women with disordered swallowing. Clin Radiol 40(4): 397-400.

7. Lopez-Castilla J, Cano M, Munoz M, Soult JA, Andrés A, et al. (1997) Massive bronchoalveolar aspiration of barium sulphate during a radiologic study of the upper digestive tract. Pediatr Pulmonol 24(2): 126-7.

8. Franquet T, Gimenez A, Roson N, Torrubia S, Sabate JM, et al. (2000) Aspiration diseases: Findings, pitfalls, and differential diagnosis. Radiographics 20(3): 673-85.

9. Taman I, Kortsik C (1999) Severe barium sulphate aspiration in the lung: clinical presentation, prognosis and therapy. Respiration 66(1): 81-84.

10. Whiting J (2003) Aspiration of barium. N Engl J Med 348(25): 2582-3.

11. Wani B, Yeola M (2008) Aspiration of barium sulphate in swallow study. Internet J Pulm Med 10(2).

12. Zhonghua Wai Ke Za Zhi (1996) Extirpation of bronchi - experimental study. 34(3): 180-181. 
ISSN: 2574-1241

DOI: $10.26717 /$ BJSTR.2021.37.006033

Yanko Pahnev. Biomed J Sci \& Tech Res

(c) (P) This work is licensed under Creative

Submission Link: https://biomedres.us/submit-manuscript.php

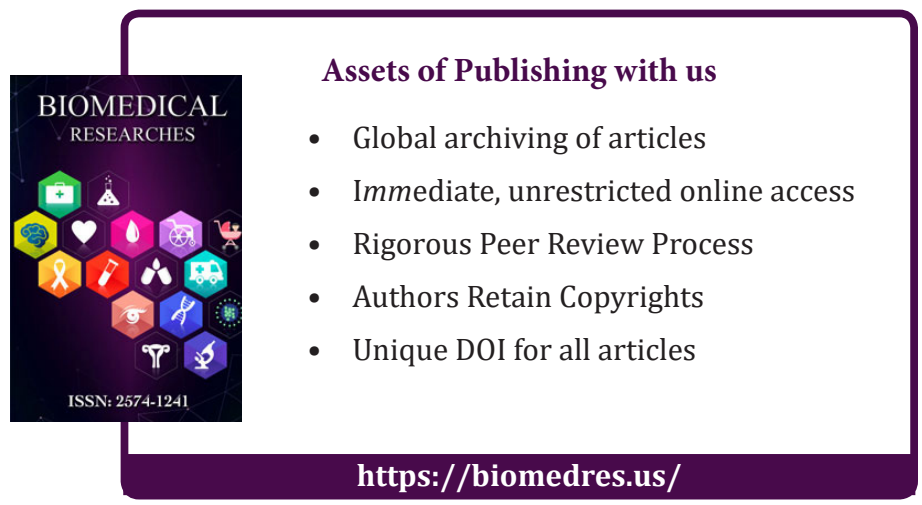

